\title{
Reciprocal Chromosome Translocation Between the Left-End 220kb of Chromosome II and the Right-End 270kb of Chromosome X in Saccharomyces cerevisiae
}

\author{
Masaharu Takeda ${ }^{1,2,3} \&$ Takahito Okushiba ${ }^{1}$ \\ ${ }^{1}$ Department of Applied Life Science, Sojo University, 4-22-1 Ikeda, Kumamoto 860-0082, Japan \\ ${ }^{2}$ Department of Materials and Biological Engineering, Tsuruoka, National College of Technology, Yamagata \\ 997-8511, Japan \\ ${ }^{3}$ Laboratory of Life Information Science, 2-18-23 Nakasakurada, Yamagata 990-2422, Japan \\ Correspondence: Masaharu Takeda, Department of Applied Life Science, Sojo University, 4-22-1 Ikeda, \\ Kumamoto 860-0082, Japan. E-mail: takedamasaharu@gmail.com
}

Received: June 8, 2018

Accepted: June 20, 2018

Online Published: July 3, 2018

doi:10.5539/ijb.v10n4p1

URL: https://doi.org/10.5539/ijb.v10n4p1

\begin{abstract}
Southern hybridization of chromosomes and the physical mapping of the genes used as several probes on the respective chromosomes II and X showed that the left-end ca. $220 \mathrm{~kb}$ of chromosome II including ATP1 was exchanged the right-end ca. $270 \mathrm{~kb}$ of chromosome $\mathrm{X}$ including $A T P 2$ resulting the reciprocal chromosome translocation in the yeast strain YNN290, Saccharomyces cerevisiae. YTO290, the mutated strain by the reciprocal chromosome translocation as above described, was changed from red to white of the colony-color, and sizes of chromosome II lengthened from ca. $830 \mathrm{~kb}$ to ca. $900 \mathrm{~kb}$ and chromosome X shortened from ca. $760 \mathrm{~kb}$ to ca. $690 \mathrm{~kb}$, respectively, in compared with the original strain YNN290. But, YTO290 strain was the same as the original strain YNN290 for other properties; the nutrient requiring of the genotype, the ploidy, the mitochondrial respiratory activity, the cell-size, and the growth-rate (doubling time), the number of chromosomes in a cell, It should be as a total number of nucleotides (bases) of genome.
\end{abstract}

ATP1 or ATP2 and their neighboring base sequences respectively should be transferred from chromosome II left-end ca. $220 \mathrm{~kb}$ to chromosome X right-end or chromosome X right-end ca. $270 \mathrm{~kb}$ to chromosome II left-end accompanying with this reciprocal chromosome translocation. This mutated (the reciprocal chromosomes II and X translocation $=$ exchanged those end-sequences as above described) strain, YTO290, seemed to lead to decrease the stability of the changed chromosomes II and X. The mutated strain, YTO290 might be observed to go back to the respective chromosomes II and X of the original strain, YNN290, in several months later even at $4^{\circ} \mathrm{C}$.

Keywords: reciprocal chromosome translocation, Saccharomyces cerevisiae, chromosome II, chromosome X, ATP1, ATP2, ade2-mutation, colony color assay, TYA- and TYB-elements, sequence spectrum method (SSM)

\section{Introduction}

With the rapid progress of the genome-projects for many prokaryotic- and eukaryotic cells, the whole-genome analyses of the biological phenomena for individual cells were carried out to understand the organisms (Takeda, 2014).

Mitochondrial ATP synthase (F1F0-ATPase complex) functions as a key enzyme for ATP production in eukaryotic cells (Cox et al., 1992). In the yeast Saccharomyces cerevisiae, ATP1 (Takeda et al., 1986) and ATP2 (Takeda et al., 1985) were respectively genes for $\alpha$ - and $\beta$-subunits of the core components of the catalytic domain, F1-portion of F1F0-ATPase complex. These two genes, ATP1 and ATP2 were nearly located the left end $(37,045-38,679$, YBL099w) (Saccharomyces Genome Database) of chromosome II and the right end (647,296-648,828, YJR121w) (Saccharomyces Genome Database) of chromosome X, respectively, and should be expressed.

The reciprocal chromosome translocation is a drastic change of plural chromosomes which a part of one chromosome was exchanged to a part of another chromosome each other, and resulted in the strain to be composed of two newly-chimera-chromosomes. Therefore, the reciprocal chromosome translocation should be reported in higher organisms, especially Homo sapiens, because most of them were accelerated the chromosomal 
instability, and related to sick, cancer and some immunological phenomena which might be observable and participated to each medical care (Neuberger \& Calabi, 1983; Showe \& Croce, 1987; Adams \& Corry, 1991; Sawyer et al., 1992; Zhang et al., 2003). But, the molecular biological and genetic analyses of the reciprocal chromosome translocation might not be so progressed to advance on the analysis of the genome-level because of the large scale for the chromosomes in higher organisms.

S. cerevisiae was a single eukaryotic cell, and each chromosome, non-coding regions and genes were small with few introns, and could be emerged of $S$. cerevisiae chromosomes. Therefore, the study using the system of $S$. cerevisiae might be straight-forward for the metabolic data and the heritable information to analyze to reflect the genome-level for the eukaryotic biological events (Schar, 2001; Kolodner et al., 2002; Theis et al., 2007). Moreover, the ade2-mutant in yeast S. cerevisiae also might be a heritable and the red/white colony color assay might an analytical tool for the distinction of the reciprocal chromosome translocation on genome-scale (Ugolini \& Bruschi, 1996).

When the molecular mechanism of the reciprocal chromosome translocation could be expounded, it would be inestimable when there might be found a good example for the reciprocal chromosome translocation in $S$. cerevisiae. Another to say, the study of the heritable instability might be an important factor for the cue of cancer, $S$. cerevisiae might be one of the most proper systems to do the molecular biological search on genome-level.

The reciprocal chromosome translocation of the yeast $S$. cerevisiae was unexpectedly found a mutated strain with different sizes of chromosomes II and $\mathrm{X}$ in a process of the chromosome fragmentation experiments (Vollrath et al., 1988) for the gene organization study of the F1F0-ATP complex using the diploid strain YNN290 (a/ $\alpha$, ade2-101/ade2-101, lys2-801/lys2-801, ura3-52/ura3-52, trp1- $\Delta 1 / \operatorname{trp} 1-\Delta 1)$, designated as the mutated strain YTO290.

The red/white colony color assay in the yeast $S$. cerevisiae has been reported as epistatic growth advantage of ade8-18, ade2 cells over red ade2 cells (Ugolini \& Bruschi, 1996). That is, in the yeast $S$. cerevisiae, the ade2-mutant should be essentially shown as a red colony in YPD and SD-selected medium, but in the presence of the suppressive gene or the suppressive conditions against the ade2, the red colony might be reduced the red-color. In this article, the ade2-mutant in yeast $S$. cerevisiae also might be a heritable and analytical tool for the reciprocal chromosome translocation on genome-scale.

\section{Materials and Methods}

\subsection{Yeast Strains}

The yeast strains used in this study were YNN290 (a/ $\alpha$, ade2-101/ade2-101, lys2-801/lys2-801, ura3-52/ura3-52,

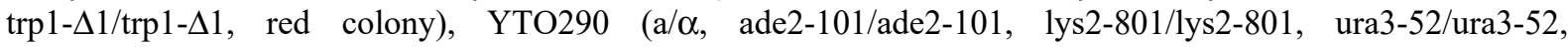

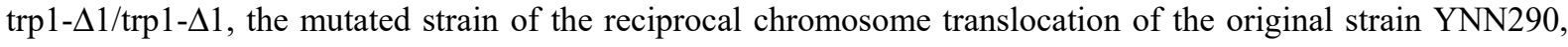
white colony on YPD, Table1 in this article), SH964 [a, ade2-101, lys2-801, ura3-52, his7, CFVII (RAD2, D)] (Takeda et al., 1994; 1995; 1999; 2010).

Table 1. Characteristic of YNN290 and YTO290

\begin{tabular}{lllllllll}
\hline $\begin{array}{l}\text { Strain } \\
\text { Medium }\end{array}$ & Colony color & Growth (doubling time) & & & & \\
& & YPD & & & SD & & YPG \\
\hline & & & complete & minus adenine & minus lysine & minus tryptopan & minus urasil \\
YNN290 & Red & 100 min & grow & not grow & not grow & not grow & not grow & grow \\
YTO290 & White & 94 min & grow & not grow & not grow & not grow & not grow & grow \\
\hline
\end{tabular}

\subsection{Vectors}

The chromosome fragmentation vectors YCF3 and YCF4 (Vollrath et al., 1988, a gift from Dr. P. Hieter) were used for ATP1 (Takeda et al., 1994; 1995, chromosome II) and ATP2 (Takeda et al., 1994; 2005, chromosome $\mathrm{X}$ ), respectively. The preparation of selective (SD) and rich (YPD and YPG(E) media (Sherman et al., 1986), and yeast transformation (Hill et al., 1991) were performed as described.

\subsection{Media}

The preparation of selective (SD) and rich (YPD and YPG(E) media (Sherman et al., 1986) and yeast transformation (Hill et al., 1991) were performed as described. 


\subsection{Colony Color Assay (Ugolini \& Bruschi, 1996)}

SD medium was containing $0.7 \%$ yeast nitrogen base w/o amino acid was added amino acid (final $20 \sim 30 \mathrm{mg}$ each amino acid) and adenine (final $6 \mathrm{mg}$ ), and agar. The red or white colonies were cropped the plate, and grow for $24 \sim 48 \mathrm{~h}$ at $28 \sim 30^{\circ} \mathrm{C}$, and distinguished the colony-color.

2.5 Chromosome Isolation by a CHEF Apparatus (Takeda et al., 1994; 1995; 1999; 2010).

Chromosomes were isolated from the strains SH964, YNN290, and YTO290, respectively. Chromosomes were isolated the strains SH964, YNN290 and YTO290, and then subjected to 1\% agarose gel electrophoresis. Chromosomes were isolated and applied to a CHEF apparatus. Electrophoresis was carried out for $34 \mathrm{~h}$ with a switching interval of $55 \mathrm{~s}$ for $17 \mathrm{~h}$ and $90 \mathrm{~s}$ for $17 \mathrm{~h}$.

\subsection{Southern Hybridization (Takeda et al., 1994; 1995; 1999; 2005; 2010)}

After the electrophoresis, Southern hybridization was carried out as described using DIG (digoxigenin)-labeled $A T P l$ and other genes as probes (Takeda et al., 1994; 1995; 1999; 2005; 2010). Preparation of DIG-labeled genes (probes) was performed as described (Takeda et al., 1994; 1995; 1999; 2005; 2010).

The intensities of the hybridized chromosomes were measured with a Densitograph.

2.7 Homologous Region of the Sequence Spectra of Chromosomes II and X (Takeda \& Nakahara, 2009; Nakahara \& Takeda, 2010)

Sequence spectra of chromosome II $(813,131 \mathrm{nt}$, SGD-data) and chromosome X (745,431nt, SGD-data) were drawn and HF (homology factor) was calculated by previously published (Takeda \& Nakahara, 2009; Nakahara \& Takeda, 2010).

\section{Results and Discussion}

\subsection{Reciprocal Chromosome Translocation between Chromosome II and Chromosome X in Saccharomyces cerevisiae}

Authors showed that the left-end ca. $220 \mathrm{~kb}$ of chromosome II including ATP1 was exchanged the right-end ca. $270 \mathrm{~kb}$ of chromosome $\mathrm{X}$ including ATP2 resulting the reciprocal chromosome translocation in Saccharomyces cerevisiae. YTO290 occurred strain above described was the same to the nutrient requiring of the original strain YNN290 (a/ $\alpha$, ade2-101/ade2-101, lys2-801/lys2-801, ura3-52/ura3-52, trp1- $\Delta 1 / \operatorname{trp} 1-\Delta 1)$, the genotype, the ploidy, the mitochondrial respiration, and the growth-rate, the number of chromosomes in a cell, total number of nucleotides (bases) of genome except the colony-color (Table 1). Although YNN290 showed red-colony on glucose-containing medium on both YPD and SD + adenine + lysine + tryptophan + uracil, YTO290 showed white-colony on above medium. Moreover, both TYA- and the TYB-elements (transposons, SGD-data, also show and discuss in Figure 6) were located within the left-end of chromosome II and within the right-end of chromosome $\mathrm{X}$, which was extremely close to each chromosome-translocation-site. ATP1 or ATP2 and their neighboring base sequences respectively should be transferred from chromosome II left-end ca. $220 \mathrm{~kb}$ to chromosome X right-arm or chromosome X right-end ca. 270kb to chromosome II left-arm accompanying with this reciprocal chromosome translocation (Figure 1). ADE2 (ade2) gene was still present in chromosome XV the same as YNN290 (data not shown). In this study, the color-color of YTO290 was changed to white the same as those of chromosome fragmentation irrespective without the plasmid containing SUP11. Each chromosome of YTO290 except chromosomes II and X showed apparently the same sequence spectra as YNN290 (data not shown). The reciprocal chromosomal translocation might be caused by some conformational change of chromosome(s) cushioned the ade2-ochre mutation, and reduced the red-color of the colony.

\subsection{Colony Color Assay}

YNN290 strain used in this study was a diploid and ade2-mutant (a/ $\alpha$, ade2-101/ade2-101, lys2-801/lys2-801, ura3-52/ura3-52, trp1- $\Delta 1 / \operatorname{trp} 1-\Delta 1$ ), and showed red-colony in YPD or SD-selected medium (Figure 2). However, when YNN290 was kept in cold room $\left(4^{\circ} \mathrm{C}\right)$, most of them showed still red-colonies on glucose-containing medium (Figure 2, plate 1and 4, upper-panel, left), but some of the colonies were changed to show white (Figure 2, plates $1 \& 4$, upper-panel, right). The ade2-mutants which showed red-color in the SD-medium restricted adenine content $(6 \mathrm{mg} / \mathrm{l})$. However, SUP11 in the plasmids (Figure 3) using chromosome fragmentation was repressed the red-color in the ade2-mutant and the colony should be showed white on glucose-containing medium (Figure 2, plates 1, 3 and 4, lower-panel). YTO290 was not grown on SD medium lacked URA3 containing the fragmentation plasmids (Figure 2, plate 3, upper-panels), therefore, YTO290 was shown to be produced by the reciprocal chromosome translocation, not by the chromosome fragmentation used plasmids, containing the suppressor against the ade2-mutant, SUP11 gene (Vollrath et al, 1988; Takeda et al., 1994; 1995; 
1999, 2005) (Figure 2, plates 1, 3 and 4, upper-panel; lower-panel, left, chromosome fragmentation of chr. X for $A T P 2$, and right, of chr. II for $A T P 1$, respectively, using the SUP11 containing plasmid).
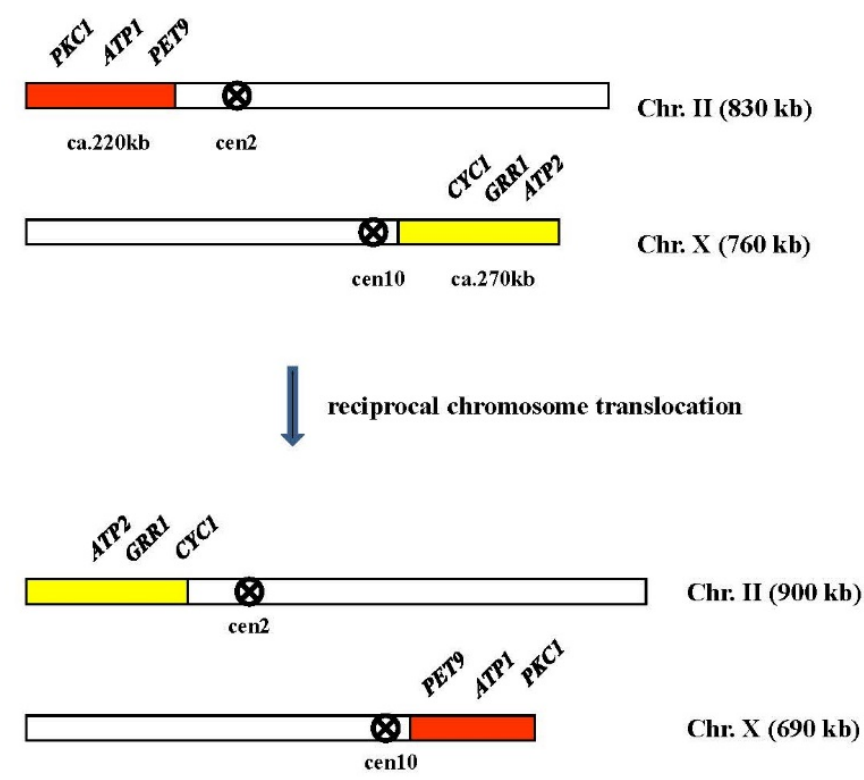

Figure 1. Reciprocal chromosome translocation in this study

Upper-panel: the original chromosomes (strain YNN290): chromosome II (ca. 830kb) and chromosome X (ca. 760kb). Lower-panel: the strain YTO290 produced from the reciprocal chromosome translocation chromosome II (ca. 900kb) and chromosome X (ca. 690kb). Chromosome II was changed from $830 \mathrm{~kb}$ to $900 \mathrm{~kb}$, and chromosome X from $760 \mathrm{~kb}$ to $690 \mathrm{~kb}$, respectively. Other 14 chromosomes were not changed.

The red-region of the chromosomes II (ca. 220kb) and the yellow-region of the chromosome X (ca. 270kb) were interchanged by the reciprocal chromosome translocation, and produced the strain YTO290) with newly chimera-chromosomes II (ca. 900kb) and X (ca. 690kb) translocation, strain YTO290): chromosome II (ca. $900 \mathrm{~kb}$ ) and chromosome X (ca. 690kb). The centromere locus $(\boldsymbol{\otimes}$, cen) of the chromosomes II (cen2) and X (cen10) were remained the original chromosomes, respectively, in this reciprocal chromosome translocation.

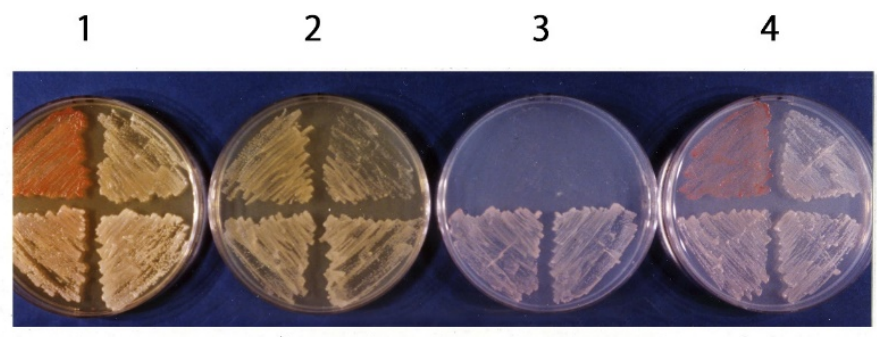

Figure 2. Red/white colony color assay of YNN290 and YTO290.

Plate 1, YPD medium; plate 2, YPG medium; plate 3, SD + ade + lys + trp; plate 4, SD + ade + lys + trp + ura. Each colony (plate) was grown at $29-30^{\circ} \mathrm{C}$ for $24-48 \mathrm{~h}$, and then conserved in cold room $\left(4^{\circ} \mathrm{C}\right)$. The strains of each plate (plates $1 \sim 4$ ) were as follows:

Upper-panel left, YNN290 (wild-type); right, YTO290.

Lower-panel left, TOY0422 (chromosome X fragmentation for ATP2, Takeda et al., 1994; 2005); right, TOY0411 (chromosome II fragmentation for ATP1, Takeda et al., 1994; 1995). 

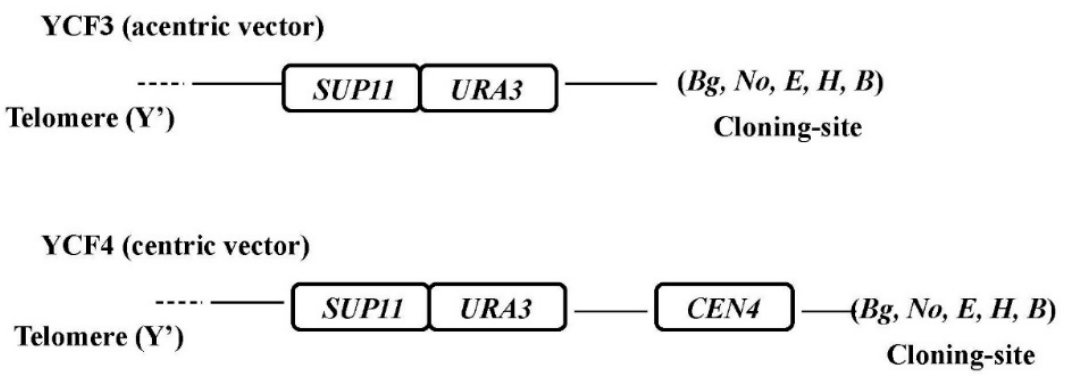

Figure 3. Plasmids using chromosome fragmentation (Takeda et al., 1994; 1995; 1999)

Upper, YCF3 (acentric vector, 7.1kb); lower YCF4 (centric vector, $8.5 \mathrm{~kb}$ ) were gifted from Dr. P. Hieter. The chromosome fragmentation plasmids YCF3 and YCF4 contained Y' (telomere), SUP11 (suppressor) and URA3 genes. YCF4 plasmid had cen4, but YCF3 did not have cen4. The cloning site of the plasmids, Bg (BglII), No (Not I), E (EcoRI), H (HindIII), B (BamHI).

These chromosome fragmentation vectors were used for the characterization, identification of the copy numbers for each nuclear-coded subunit gene of $\mathrm{F} 1 \mathrm{~F} 0$-ATPase complex in Saccharomyces cerevisiae chromosomes for ATP1 (chr.II), ATP2 (chr.X), ATP3 (chr. II), ATP4 (chr.XVI), ATP5 (chr.XVI), ATP7 (chr.XI), ATP14 (chr.IV), $A T P 15$ (chr.XVI), ATP16 (chr.IV) (data not shown for each gene).

Surprisingly, when YTO290 was kept in cold room $\left(4^{\circ} \mathrm{C}\right)$ again, they had changed to show red so as to the original strain, YNN290. That is, YTO290 went back the red-colonies on glucose-containing medium. The YTO290 might be decreased the stability of the fragment by somehow the conformational change, therefore, in related to the reciprocal chromosome-translocation might be re-occurred. This second reciprocal chromosome translocation (the mutated strain YTO290 went back to the original strain YNN290) might be accelerated to the genomic instability and cancer when DNA replication should be escaped control (Sawyer et al., 1992; Schar, 2001; Kolodner et al., 2002; Zhang et al., 2003).

The red/white colony color assay in the yeast $S$. cerevisiae has been reported as epistatic growth advantage of ade8-18, ade2 cells over red ade2 cells (Ugolini \& Bruschi, 1996; Theis et al., 2007). That is, in the yeast $S$. cerevisiae, strain YNN290, the ade2-mutant, should be essentially shown as a red colony on YPD or SD-selected medium, but the red color of the colony should be reduced by the presence of the suppressor gene or the suppressive conditions against the ade2-mutant. As results, when the reciprocal chromosome translocation has occurred, the colonies became white (strain YTO290) was observed in a plate. But, the strain YTO290 might be unstable and gone back to the original strain YNN290 because the suppressive conditions against the ade2-mutant might be disappeared.

\subsection{Southern Hybridization}

To check the chromosomes of strain of the red- and the white-colonies by the southern blot hybridization with $A T P 1, A T P 2$ and other genes as DIG-labeled probes. After the pulsed field gel electrophoresis, the gel was blotted to membrane and hybridized with some DIG-labeled probes, ATP2, GRR1, CYC1, CYR1 and other genes resided in chromosome X (Figure 4A, 4B), and ATP1, LYS2, PHO3/5, SUP45, CDC28 and other genes resided in chromosome II (Figure 4C, 4D).

As shown in Figure 4, the red colonies were the same chromosomal pattern as the original strain YNN290 (same as the haploid strain SH964, Figure 4A-D, lane 1, Figure 4A, lane $2=$ YNN290), but the white colonies, YTO290 were changed the chromosomal pattern (Figure 4A, 4C and 4D, lanes 3 and 4). The results of the genes from the southern blot hybridization were shown in Table 2 and Figure 5. That is, the reciprocal chromosome translocation in yeast $S$. cerevisiae was occurred between chromosome II left-end, ca. 220kb containing ATP1 gene and chromosome $\mathrm{X}$ right-end, ca. 270kb containing ATP2 gene. As results, the red-colony of the original strain YNN290 was changed to the white-colony of the strain YTO290 on YPD or SD + ade + lys + trp + ura medium so as to the ade2-mutant transformed irrespective without the SUP11-containing chromosome fragmentation plasmids. Another to say, this red/white colony color change could be occurred by the reciprocal chromosome translocation without using the chromosome-fragmentation plasmid contained SUP11 gene. 


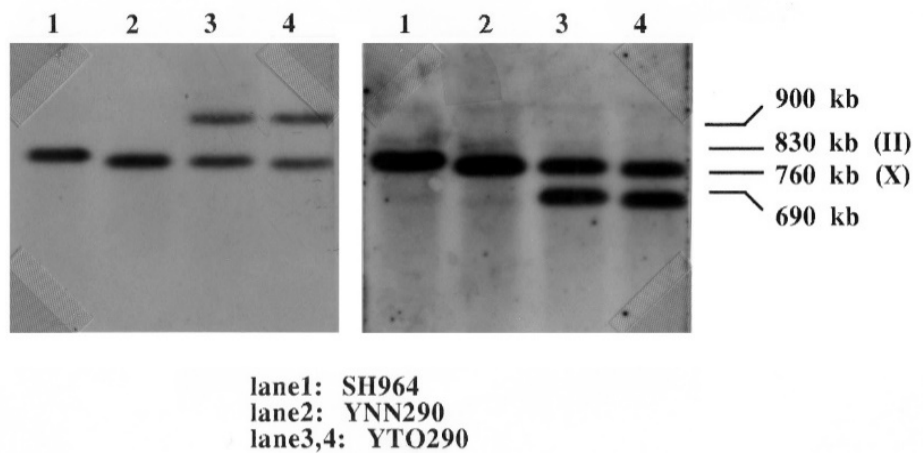

(C) ATP1, PET9(AAC2)

(D) fur4, LYS2, PHO3/5, SUP45, CDC28
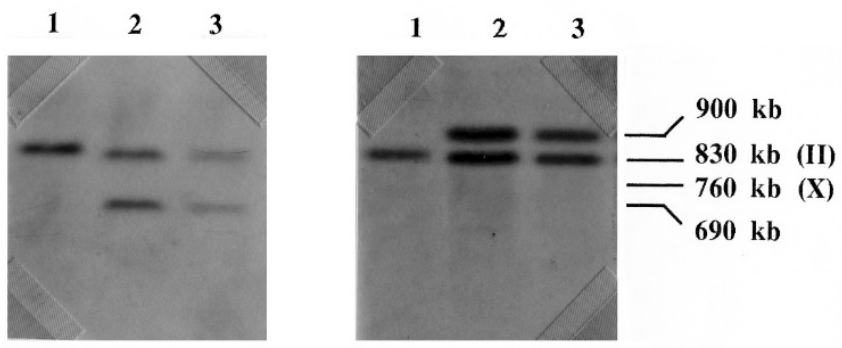

\section{lane1: SH964}

lane2,3: YTO290

Figure 4. Southern hybridization

After the isolation of S. cerevisiae (strain, SH964, YNN290, YTO290) chromosomes by electrophoresis with a CHEF apparatus, southern hybridization was carried out as described using DIG(digoxigenin)-labeled $A T P l$ and other genes as probes (Takeda et al., 1994; 1995; 1999). Preparation of DIG-labeled genes (probes) was performed as described by. Takeda et al., 1994; 1995; 1999. (A) \& (B), lane 1: SH964; lane 2: YNN290; lanes 3, 4: YTO290. (C) \& (D), lane 1: SH964; lanes 2, 3: YTO290.

(A) probe: ATP2, GRR1, CYC1; (B) probe: CYR1; (C) probe: ATP1, PET9 (AAC2) ; (D) probe: FUR4, LYS2, $P H O 3 / 5, S U P 45, C D C 28$ and so on.
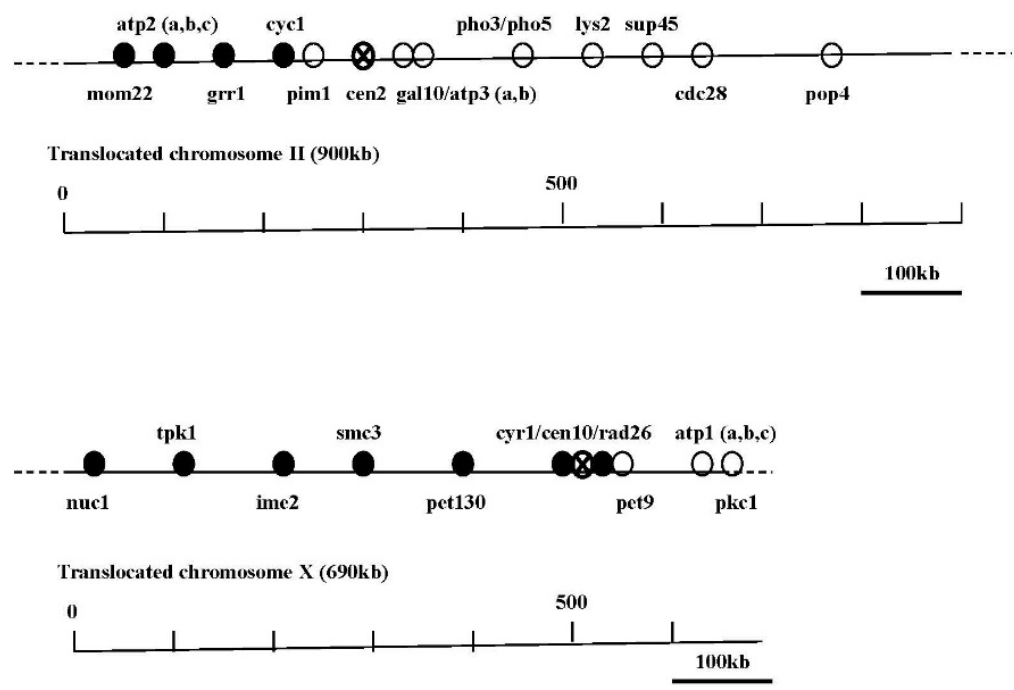

Figure 5. Physical mapping of the genes in the newly synthesized chimera-chromosomes II in and X in this reciprocal chromosome translocation 
Upper the arrow-head original chromosomes (strain YNN290): chromosome II (ca. 830kb) and chromosome X (ca. 760kb). Lower the arrow-head (after reciprocal chromosomes II and X translocation, strain YTO290): chromosome II (ca. $900 \mathrm{~kb}$ ) and chromosome X (ca. 690kb). The centromere locus (cen) of the chromosomes II and $\mathrm{X}$ were remained the original chromosomes, respectively, in this reciprocal chromosome translocation. Gene (chr.II, O): pkc1 (= ser/thr protein kinase. PKC1), atp1 (= F1F0-ATPase complex, F1 alpha subunit. ATP1), pet9 (= ADP/ATP carrier protein. PET9), pim1 (= ATP-dependent protease, mitochondria. PIM1), cen2 $(=$ centromere2. cen2), gal10 (= UDP-glucose 4-epimerase. GAL10), atp3 (= F1F0-ATPase complex, F1 gamma subunit. ATP3), pho3/5 (= constitutive acid phosphatase precursor, $P H O 3 /$ repressible acid phosphatase precursor, PHO5), lys2 (= L-aminoadipate-semialdehyde dehydrogenase large subunit. LYS2), sup45 (= translational release factor. SUP45), cdc28 (= cyclin-dependent protein kinase. CDC28), pop4 (= involved in processing of tRNAs and rRNAs. POP4) over the genes in original (YNN290) chromosome II. Gene (chr. X, O): nuc1 (= nuclease, mitochondria. NUC1), tpk1 (= cAMP-dependent protein kinase. catalytic chain. TPK1), ime2 (= ser/thr protein kinase. IME2), smc3 (= required for structural maintenance of chromosomes. SMC3), pet130 (= protein synthesis protein, mitochondria. PET130), cyr1 (= adenylate cyclase, CYRI), cen10 (= centromere10, cen10), $\operatorname{rad} 26$ (= DNA repair and recombination protein. RAD26), cyc1 (= cytochrome c isoform 1, CYC1), sup4 (= tRNA-Tyr, SUP4), hoc1 (= suppressor of pkc1, HOC1), grr1 (= required for glucose repression and for glucose and cation transport, GRR1), atp2 (= F1F0-ATPase complex, F1 beta subunit, ATP2), mom22 (= required for maintenance of chromosomes and mini-chromosomes, MOM22) over the original (YNN290) chromosome X. The arrow head for each chromosome might be the possible reciprocal chromosome translocation-site.

Table 2. Southern blot hybridization

\begin{tabular}{|c|c|c|c|c|c|c|c|c|c|c|}
\hline & (lane 1) & (lane 2) & (lane 3) & & & & (lane 4) & & & \\
\hline Strain & SH964 & YNN290 & YTO290 & & & & YTO290 & & & \\
\hline MAT & a & $\mathrm{a} / \alpha$ & $\mathrm{a} / \alpha$ & & & & $\mathrm{a} / \alpha$ & & & \\
\hline Probe (gene) & \multicolumn{10}{|c|}{ Size of chromosome hybridized with the probe } \\
\hline (A)ATP2 & $760 \mathrm{~kb}$ & $760 \mathrm{~kb}$ & $900 \mathrm{~kb}$ & & $760 \mathrm{~kb}$ & & $900 \mathrm{~kb}$ & & $760 \mathrm{~kb}$ & \\
\hline GRR1 & $760 \mathrm{~kb}$ & $760 \mathrm{~kb}$ & $900 \mathrm{~kb}$ & & $760 \mathrm{~kb}$ & & $900 \mathrm{~kb}$ & & $760 \mathrm{~kb}$ & \\
\hline $\mathrm{CYC1}$ & $760 \mathrm{~kb}$ & $760 \mathrm{~kb}$ & $900 \mathrm{~kb}$ & & $760 \mathrm{~kb}$ & & $900 \mathrm{~kb}$ & & $760 \mathrm{~kb}$ & \\
\hline CDC11 & $760 \mathrm{~kb}$ & $760 \mathrm{~kb}$ & $900 \mathrm{~kb}$ & & $760 \mathrm{~kb}$ & & $900 \mathrm{~kb}$ & & $760 \mathrm{~kb}$ & \\
\hline \multicolumn{11}{|l|}{ SUP4 } \\
\hline (B)CYR1 & $760 \mathrm{~kb}$ & & & & $760 \mathrm{~kb}$ & $690 \mathrm{~kb}$ & & & $760 \mathrm{~kb}$ & $690 \mathrm{~kb}$ \\
\hline (C)ATP1 & $830 \mathrm{~kb}$ & & & $830 \mathrm{~kb}$ & & $690 \mathrm{~kb}$ & & $830 \mathrm{~kb}$ & & $690 \mathrm{~kb}$ \\
\hline $\mathrm{PKC1}$ & $830 \mathrm{~kb}$ & & & $830 \mathrm{~kb}$ & & $690 \mathrm{~kb}$ & & $830 \mathrm{~kb}$ & & $690 \mathrm{~kb}$ \\
\hline $\mathrm{CDC} 27$ & $830 \mathrm{~kb}$ & & & $830 \mathrm{~kb}$ & & $690 \mathrm{~kb}$ & & $830 \mathrm{~kb}$ & & $690 \mathrm{~kb}$ \\
\hline ILS1 & $830 \mathrm{~kb}$ & & & $830 \mathrm{~kb}$ & & $690 \mathrm{~kb}$ & & $830 \mathrm{~kb}$ & & $690 \mathrm{~kb}$ \\
\hline PET9 & $830 \mathrm{~kb}$ & & & $830 \mathrm{~kb}$ & & $690 \mathrm{~kb}$ & & $830 \mathrm{~kb}$ & & $690 \mathrm{~kb}$ \\
\hline (D)LYS2 & $830 \mathrm{~kb}$ & & $900 \mathrm{~kb}$ & $830 \mathrm{~kb}$ & & & $900 \mathrm{~kb}$ & $830 \mathrm{~kb}$ & & \\
\hline PHO3/5 & $830 \mathrm{~kb}$ & & $900 \mathrm{~kb}$ & $830 \mathrm{~kb}$ & & & $900 \mathrm{~kb}$ & $830 \mathrm{~kb}$ & & \\
\hline SUP45 & $830 \mathrm{~kb}$ & & $900 \mathrm{~kb}$ & $830 \mathrm{~kb}$ & & & $900 \mathrm{~kb}$ & $830 \mathrm{~kb}$ & & \\
\hline CDC28 & $830 \mathrm{~kb}$ & & $900 \mathrm{~kb}$ & $830 \mathrm{~kb}$ & & & $900 \mathrm{~kb}$ & $830 \mathrm{~kb}$ & & \\
\hline
\end{tabular}

Recently, we have found three identical ATP1 genes on chromosome II designated as ATP1a, ATP1b, ATP1c were repeated tandem with the neighboring DNA sequence (ca. 16kb, Takeda et al., 2010) on the left-hand of chromosome II in S288C, DC5, W303-1A strains of S. cerevisiae (Takeda et al., 1995; 1999; 2010). Similarly, three identical ATP2 genes on chromosome X (designated as ATP2a, ATP2b, ATP2c. Takeda et al., 2005) was arranged in tandem with the neighboring sequence (ca. 14kb) on the right-hand chromosome $\mathrm{X}$ in $S$. cerevisiae (Takeda et al., 2005). The results of the southern hybridization using ATP1 or ATP2 as probes, after careful thought, these three ATP1 genes or ATP2 genes were respectively. It should be identical, and might be also respectively transferred together to the right-arm of the chromosome $\mathrm{X}$, and to the left-arm of the chromosome II in the reciprocal chromosome translocation

In this article, the reciprocal chromosome translocation in the yeast $S$. cerevisiae was occurred between chromosome II left-end, ca. 220kb containing three identical ATP1 genes and chromosome X right-end, ca. $270 \mathrm{~kb}$ containing three identical ATP2 genes, in addition, the resulted strain YTO290 was changed to the white-colony from the red-colony of the original strain YNN290 cultured in YPD or SD + ade + lys + trp + ura 
medium so as to the ade2-mutant transformed with the SUP11-containing chromosome fragmentation plasmids, YCF3 (acentric, 7.1kb) and YCF4 (centric, $8.5 \mathrm{~kb}$ ) as above described. Table 2 showed several characters of the genes resided in chromosomes II and X for both YNN290 and YTO290. These two strains were almost the same grown on YPG, YPD and SD selected media, doubling-time and other growth conditions except the colony-color. The red/white colony color assay in the yeast $S$. cerevisiae has been reported as epistatic growth advantage of ade8-18, ade2 cells over red ade2 cells (Ugolini \& Bruschi, 1996). That is, in the yeast $S$. cerevisiae, the ade2-mutant should be essentially shown red colony in YPD and SD-selected medium, but in the presence of the suppressive gene or condition(s) against the $a d e 2$, the red colony might be reduced the red-color. Again we have not used with the SUP11-containing chromosome fragmentation plasmids in this study, but YTO290, strain obtained by the reciprocal chromosomal translocation was reduced the red-color of YNN290 strain of the ade2-mutation. The reciprocal chromosome translocation between the chromosome II left-end and the chromosome $\mathrm{X}$ right-end might be occurred in some way suppressive effects against the ade2-mutation accompanying conformational change of chromosome, and decrease of the red-color of the ade2-mutatant on the chromosome-level of $S$. cerevisiae.

When YTO290 strain was kept in cold room $\left(4^{\circ} \mathrm{C}\right)$ again, the white-colonies were back to the red-colonies on glucose-containing medium (same as Figure 3, plates 1 and 4, upper-panel, left). The chromosomes were isolated from the red-colonies and checked by the southern hybridization using several probes above described, and made sure the same as them of the original strain YNN290. That is, YTO290 might be decreased the stability and gone back to the original strain YNN290 even at $4{ }^{\circ} \mathrm{C}$. Because the selection between YTO290 and YNN290 could be possible the color of colony and simple for the cultivation, and the study of the molecule- and genome-level for the reciprocal chromosome translocation will be moved what's more. The genome data showed that there might be many non-coding regions in each chromosome, genes for tRNAs and their genes controlled genes for biological information of chromosomes and cells. Some of them might be participated these suppressive effects of the chromosome conformational change. Renew, the original strain YNN290 was mutated to ade2-oucar which showed red-color in the SD-medium restricting adenine content $(6 \mathrm{mg} / \mathrm{l})$. There were many suppressive genes and tRNAs in chromosomes II, $\mathrm{X}$ and other chromosomes in S. cerevisiae. It might be possible that one of the suppressive gene(s) or conditions based on the conformational change of chromosome affected by the SUP families, SUP4 = chr.X (Kurjan \& Hall, 1982); SUP5 = chr.XIII (Piper \& Wasserstain, 1976); SUP7 = chr. X (Gilmore et al., 1971); SUP9 (BEM2 = YER155c) = chr.V (Willis et al., 1986); ADE8 (YDR408c) $=$ chr.IV (Jones \& Fink, 1982); MAF1 (YDR005c) = chr.IV (Willis et al., 1986); ADE13 (YLR359w) = chr.XII (Jones \& Fink, 1982), etc. One, or several of these tRNAs and the suppressors (proteins) might be reduced the red-color, and the colony showed white. In addition, this reciprocal chromosome translocation should be maintained not only the base-sequences of chromosomes, but also the chromosomes II- and X-ends so as to the original strain YNN290, therefore, the generation-rule and the complexity of $S$. cerevisiae-genome might be extremely similar to YNN290 (Boguta et al., 1997; Kachroo et al., 2015). Many tRNAs were existed in the non-coding regions on chromosomes, and they might be influenced to the gene expression, the gene activity, the chromosome conformation and configuration, but their role to these cellular functions might be still remained not a little unknown particular (Takeda \& Nakahara, 2009). The detailed analysis is under the progress.

\subsection{Sequence Spectra of chromosomes II and X}

The analytical method of genome structure, the sequence spectra based on the appearance frequency (SSM) might be useful to analyze the structural feature of the genomic DNA (Takeda \& Nakahara, 2009; Nakahara \& Takeda, 2010). Because the original strain YNN290 and the mutated strain YTO290 were in possession of the identical chromosomes, genes and total nucleotide-numbers (bp) of chromosomes except the sizes (nt) of chromosomes II and X for the reciprocal chromosome translocation, the appearance frequency of $S$. cerevisiae genome were used the identical value. Figure 6 showed the sequence spectra of chromosomes II and X based on the appearance frequency of three successive nucleotide sequences (triplet) in a single-strand DNA of $S$. cerevisiae entire genome. The homology factor between chromosome II and chromosome X compared with the sequence spectra. As results shown in Figure 6, the red-area of chromosome II was homologous with the dark-blue-area of chromosome $\mathrm{X}(\mathrm{HF}=$ homology factor $99.0 \%)$. The homologous areas located in 258,361-263,801 of chromosome II and in 476,601-481,911 of chromosome X, respectively, which were extremely close to the TY1A- and TY1B-elements as shown in thick arrow, respectively, and they might be also concerned with the reciprocal chromosome translocation-site in each chromosome. It could be possible that the TYA- and TYB-elements might be influenced to this reciprocal chromosome translocation. 
(A) Chr.II, $\mathrm{M}=813,131 \mathrm{nt}, \mathrm{d}=3, \mathrm{HF}=99.1 \%$.

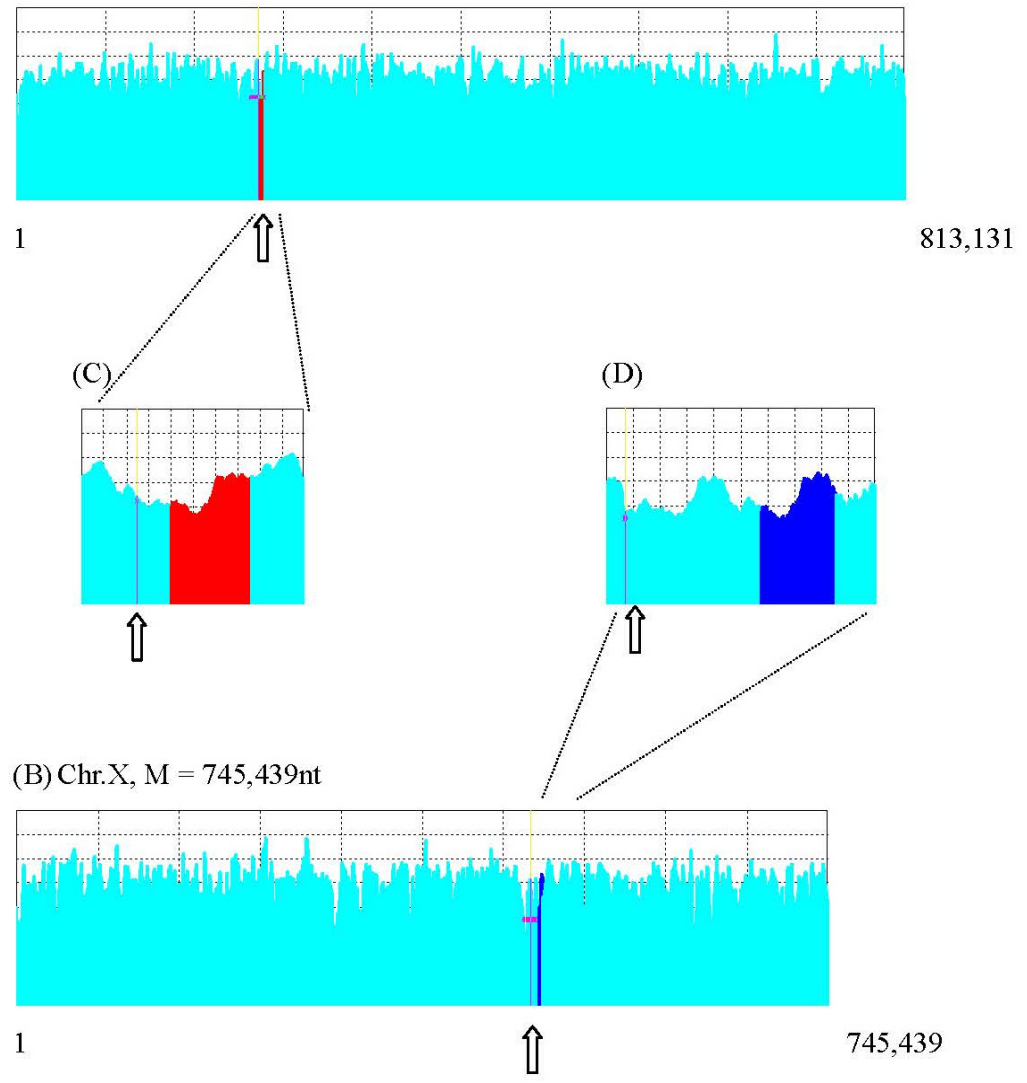

Figure 6. Comparison with the sequence spectra of chromosome II and chromosome $\mathrm{X}$

Homology of chromosome II (813131bp, SGD) with chromosome X (745431bp, SGD). Sequence spectra of the appearance frequency of chromosome II and X for the original strain YNN290 was three successive nucleotide (base) sequences (triplet) of the entire genome of $S$. cerevisiae; Nsum $=500$, Nskip $=10, d=3$ (Yeast). Nmax $=$ 100000, Ntin $=100$, Nrin $=1, \mathrm{Nwid}=400, \mathrm{Novl}=50$. Homology factor $(\mathrm{HF})$ of the homologous areas, which should be contained both TYA and TYB-elements (shown in chromosome II (red) and chromosome X (dark-blue) were $99.1 \%$. Vertical-lines (also shown the position as the thick arrow), indicated as the reciprocal chromosome translocation-site for chromosome II (ca. 220kb from left telomere), and for chromosome X (ca. $472 \mathrm{~kb}$ from the right telomere), respectively. These reciprocal chromosome translocation-sites were also shown in Figs. 1 and 5.

In the Figure 6, the horizontal (x)-axis was indicated the nucleotide-sequences (from left to right) and the vertical (y)-axis the appearance frequency of three successive nucleotide-sequences (triplet), respectively. (A), (C): YNN290 chromosome II, (B), (D): YNN290 chromosome X.

\section{Conclusion}

The author has been reported for the relationship between the genome and the biological information (Takeda, 2012). In this article, the authors could be found accidentally the reciprocal chromosome II and X translocation in the yeast $S$. cerevisiae. The reciprocal chromosome translocation could be detected the size of chromosomes II and $\mathrm{X}$ and also observed directly the color of colony (YNN290: 830kb of chr. II and chr. X, red-colony; YTO290: 900kb of chr. II and 690kb of chr. X, white-colony, respectively). The strain YTO290 might be unravelled to suppressive condition by the reciprocal chromosome translocation and should be unstable, and easily gone back to the original strain YNN290. Recently, Anderson-Lee et al were proposed the participation to the biological events for the RNA secondary structure (Anderson-Lee et al., 2016). The instability of chromosome by the reciprocal chromosome translocation might also be affected through the tRNA secondary structure (Boguta et al., 1997; Kachroo et al., 2015).

\section{Acknowledgements}

The authors wish to thank to Dr. Masatoshi Nakahara for his critical reading and advice of this article. 


\section{Authors contribution}

MT was lead researcher of the project. All authors were involved in experimental design. TO performed mainly experiments. MT analyzed the results and wrote the paper. All authors read and approved the final manuscript.

\section{References}

Adams, J. M., \& Corry, S. (1991). Transgenic mice, models of tumor development. Science, 254, 1161-1167.

Anderson-Lee, J., Fisker, E., Kosaraju, V., Wu, M., Kong, J., Lee, J., Lee, M., Zada, M., Treuille, A., \& Das, R. (2016). Principles for predicting RNA secondary structure design difficulty. J. Mol. Biol., 428, 748-757.

Boguta, M., Czerska, K., \& Zoladek, T. (1997). Mutation in a new gene MAF1 affects tRNA suppressor efficiency in Saccharomyces cerevisiae. Gene, 185, 291-296.

Cox, G. B,. Devenish, R. J., Gibson, F., Howitt, S. M., \& Nagley, P. (1992). The structure and assembly of ATP synthase. In L. Ernstar (Ed.), Molecular mechanism in bioenergetics (pp. 283-315). Amsterdam: Elsevier.

Gilmore, R. A., Stewart, J. W., \& Sherman, F. (1971). Amino acid replacements resulting from super-suppression of nonsense mutants of iso-1-cytochrome c from yeast. J. Mol. Biol., 61, 157-173.

Hill, J., Donald, K. A. I. G., \& Griffiths, D. E. (1991). DMSOenhanced whole cell yeast transformation. Nucleic Acids Res., 19, 5791.

Jones, E. W., \& Fink, G. R. (1982). Regulation of amino acid and nucleotide biosynthesis in yeast. The Molecular Biology of the Yeast Saccharomyces: Metabolism and Gene Expression, edited by Strathern, J.N., Jones, E.W. and Broach, J.R. Cold Spring Harbor Laboratory, Cold Spring Harbor, NY:181-299.

Kachroo, A. H., Laurent, J. M., Yellman, C. M., Meyer, A. G., Wilke, C. O., \& Marcotte, E. M. (2015). Evolution. Systematic humanization of yeast genes reveals conserved functions and genetic modularity. Science, 348, 921-925.

Kolodner, R. D., Putnam, C. D., \& Myung, K. (2002). Maintenance of genome stability in Saccharomyces cerevisiae. Science, 297, 565-569.

Kurjan, J., \& Hall, B. D. (1982). Mutations at the Saccharomyces cerevisiae SUP4 tRNA(Tyr) locus: Isolation, Genetic Fine-Structure Mapping, and Correlation with Physical Structure. Mol. Cell. Biol., 2, 1501-1513.

Nakahara, M., \& Takeda, M. (2010). Characterization of the sequence spectrum of DNA based on the appearance frequency of the nucleotide sequences of the genome-A new method for analysis of genome structure. J. Biomed. Sci. Eng., 3, 340-350. doi:10.4236/jbise.2010.34047. http://www.SciRP.org/journal/jbise/

Neuberger, M. S., \& Calabi, F. (1983). Reciprocal chromosome translocation between c-myc and immunoglobulin $\gamma 2 \mathrm{~b}$ genes. Nature, 305, 240-243. doi:101038/305240a0.

Piper, P. W., \& Wasserstain, M. (1976). Nonsense suppressors of Saccharomyces cerevisiae can be generated by mutation of the tyrosine tRNA anticodon. Nature, 262, 757-761.

Saccharomyces Genome Database (SGD-data).

Sawyer, J. R., Tryka, A. F., \& Lewis, J. M. (1992). A novel reciprocal chromosome translocation t (11, 22)(p13, q12) in an intraabdominal desmoplastic small round-cell tumor. Am. J. Surg. Pathol., 16, 411-416.

Schar, P. (2001). Spontaneous DNA damage, genomic instability, and cancer-when DNA replication escape control. Cell, 104, 329-332.

Sherman, F., Fink, G. R., \& Hicks, J. B. (1986). Methods Yeast Genetics. Cold Spring Harbor Laboratory, Cold Spring Harbor, NY.

Showe, L. C., \& Croce, C. M. (1987). The role of chromosomal translocations in B- and T-cell neoplasia. Ann. Rev. Immunol., 5, 253-277.

Theis, J. F., Dershowitz, A., Irene, C., Maciariello, C., Tobin, M. L., Liberi, G., Tabrizifard, S., Korus, M., Fabiani, L., \& Newlon, C. S. (2007). Identification of mutations that decrease the stability of a fragment of Saccharomyces cerevisiae chromosome III lacking efficient replications. Genetics, 177, 1445-1458.

Takeda, M. (2012). How is the biological information arranged in genome? American J. Mol. Biol., 2, 171-186. https://doi.org/10.4236/ajmb.201223019 
Takeda, M. (2014). The power-law-tail in the distribution of the nucleotides of genomes was related to the complexity of organism: New classification of organism. International J. Biol., 6, 77-96. doi:10.5539/ijb.v6n2p77 URL:http://dx.doi.org/10.5539/ijb.v6n2p77

Takeda, M, Vassarotti, A., \& Douglas, M. G. (1985). Nuclear Genes Coding the Yeast Mitochondrial Adenosine Triphosphatase Complex-primary sequence analysis of ATP2 encoding the F1-ATPase $\beta$-subunit precursor. J. Biol. Chem., 260, 15458-15465.

Takeda, M., Chen, W. -C., Saltzgaber, J., \& Douglas, M. G. (1986). Nuclear genes encoding the yeast mitochondrial ATPase complex-analysis of ATP1 coding the F1-ATPase $\alpha$-subunit and its assembly. $J$. Biol. Chem., 261, 15126-15133.

Takeda, M., Okushiba, T., Hayashida, T., \& Gunge, N. (1994). ATP1 and ATP2, the F1F0-ATPase $\alpha$ and $\beta$ subunit genes of Saccharomyces cerevisiae, are respectively located on chromosome II and X. Yeast, 10, 1531-1534.

Takeda, M., Okushiba, T., Satoh, T., Kuniyoshi, S., Morishita, C., \& Ichimura, Y. (1995). Three ATP1 genes are present on chromosome II in Saccharomyces cerevisiae. J. Biochem. (Tokyo), 118, 607-613.

Takeda, M., Satoh, H., Ohnishi, K., Satoh, T., \& Mabuchi, T. (1999). The three copies of ATP1 gene are arranged in tandem on chromosome II of the yeast Saccharomyces cerevisiae S288C. Yeast, 15, 873-878.

Takeda, M., Katayama, H., Satoh, T., \& Mabuchi, T. (2005). Three copies of the ATP2 genes are arranged in tandem on chromosome X in the yeast Saccharomyces cerevisiae. Curr. Genet., 47, 265-272.

Takeda, M., \& Nakahara, M. (2009). Structural features of the nucleotide sequences of genomes. J. Comp. Aid. Chem., 10, 38-52.

Takeda, M., Nakamura, S., \& Matsushita, S. (2010). Genome organization of the three identical ATPl genes on the left arm of chromosome II of Saccharomyces cerevisiae. Sequence analysis of the $35-\mathrm{kb}$ region containing three ATP1 genes. The Open Genomics J. (Bentham Open), 3, 9-17.

Ugolini, S., \& Bruschi, C. V. (1996). The red/white colony color assay in the yeast Saccharomyces cerevisiae: epistatic growth advantage of white ade8-18, ade2 cells over red ade2 cells. Curr. Genet., 30, 485-492.

Vollrath, D., Davis, R. W., Connelly, C., \& Hieter, P. (1988). Physical mapping of large DNA by chromosome fragmentation. Proc. Natl. Acad. Sci. U.S.A., 85, 6027-6031.

Willis, I., Frendewey, D., Nichols, M., Hottinger-Werlen, A., Schaack, J., \& Soll, D. (1986). A single base change in the intron of a serine tRNA affects the rate of RNase P cleavage in vitro and suppressor activity in vivo in Saccharomyces cerevisiae. J. Biol. Chem., 261, 5878-5885.

Zhang, P. J., Goldblum, J. R., Pawel, B. R., Fisher, C., Pasha, T. L., \& Barr, F. G. (2003). Immunophenotype of desmoplastic small round cell tumors as detected in cases with EWSWT1 gene fusion product. Med. Pathol., $16,229-235$.

\section{Copyrights}

Copyright for this article is retained by the author(s), with first publication rights granted to the journal.

This is an open-access article distributed under the terms and conditions of the Creative Commons Attribution license (http://creativecommons.org/licenses/by/4.0/). 